\title{
Chemistry and biology of asparagine-linked glycosylation*
}

\author{
Barbara Imperiali, $†$ Sarah E. O'Connor, Tamara Hendrickson and \\ Christine Kellenberger
}

\author{
Division of Chemistry and Chemical Engineering,California Institute of Technology, \\ Pasadena, CA 91125, USA
}

\begin{abstract}
The biosynthesis of glycoprotein conjugates is a complex process that involves the collective action of numerous enzymes. Recent research on the chemistry and biology of asparagine-linked glycosylation in our group has been focused on two specific areas. These are the development of potent inhibitors of oligosaccharyl transferase and the investigation of the conformational consequences of the glycosylation process. Since asparagine-linked glycosylation is an essential eukaryotic process, an understanding of the details of this complex transformation is of utmost importance both to fundamental biochemistry and to a consideration of the mechanisms of homeostatic control.
\end{abstract}

\section{INTRODUCTION}

Protein glycosylation impacts both the functional capacity and structural framework of all glycoproteins $[1,2]$. The carbohydrate modifications of proteins fall into three general categories: $N$-linked modification of asparagine [3-5], $O$-linked modification of serine or threonine [6], and glycosylphosphatidyl inositol derivatization of the $C$-terminus carboxyl group [7]. Each of these transformations is catalyzed by one or more enzymes which demonstrate different peptide sequence requirements and reaction specificities.

This paper will focus on $N$-linked glycosylation, which is the most common of the eukaryotic glycosylation reactions [4]. $N$-linked glycosylation is catalyzed by a single enzyme, oligosaccharyl transferase (OT), and involves the co-translational transfer of a lipid-linked tetradecasaccharide $\left(\mathrm{GlcNAc}_{2}-\mathrm{Man}_{9}-\mathrm{Glc}_{3}\right)$ to an asparagine side chain (in the consensus sequence Asn-Xaa-Ser/Thr) within a nascent polypeptide. The reaction is illustrated in Fig. 1. This co-translational event occurs in the endoplasmic reticulum (ER) while the polypeptide is being biosynthesized on membrane-associated ribosomes. Approximately 14 residues of the nascent peptide must clear the luminal surface of the ER membrane before oligosaccharyl transferase-mediated glycosylation can occur, thereby implying that the active site of the enzyme resides in the soluble domain of the enzyme [8]. The subsequent diversification of these conjugates arises from enzyme catalyzed processing steps that occur in the ER and Golgi apparatus after the addition of the first triantennary oligosaccharide complex (Fig. 1).

\section{The role of peptide conformation in asparagine-linked glycosylation}

Oligosaccharyl transferase glycosylates at the tripeptide recognition sequence Asn-Xaa-Thr/Ser, where Xaa represents any of the encoded amino acids except proline [9]. Because $N$-linked glycosylation occurs before the nascent polypeptide has completely folded, the native protein structure presumably does not play a role in the recognition events that lead to this modification. However, since tripeptides can act as substrates for OT, the local secondary structure of the recognition sequence and adjacent peptide may well enable recognition by the enzyme and modulate the reactivity of the amide nitrogen that acts as a nucleophile to form the protein-carbohydrate linkage.

*Lecture presented at the 19th International Carbohydrate Symposium (ICS 98), San Diego, California, 9-14 August 1998, pp. 719-800.

$\dagger$ Corresponding author. 


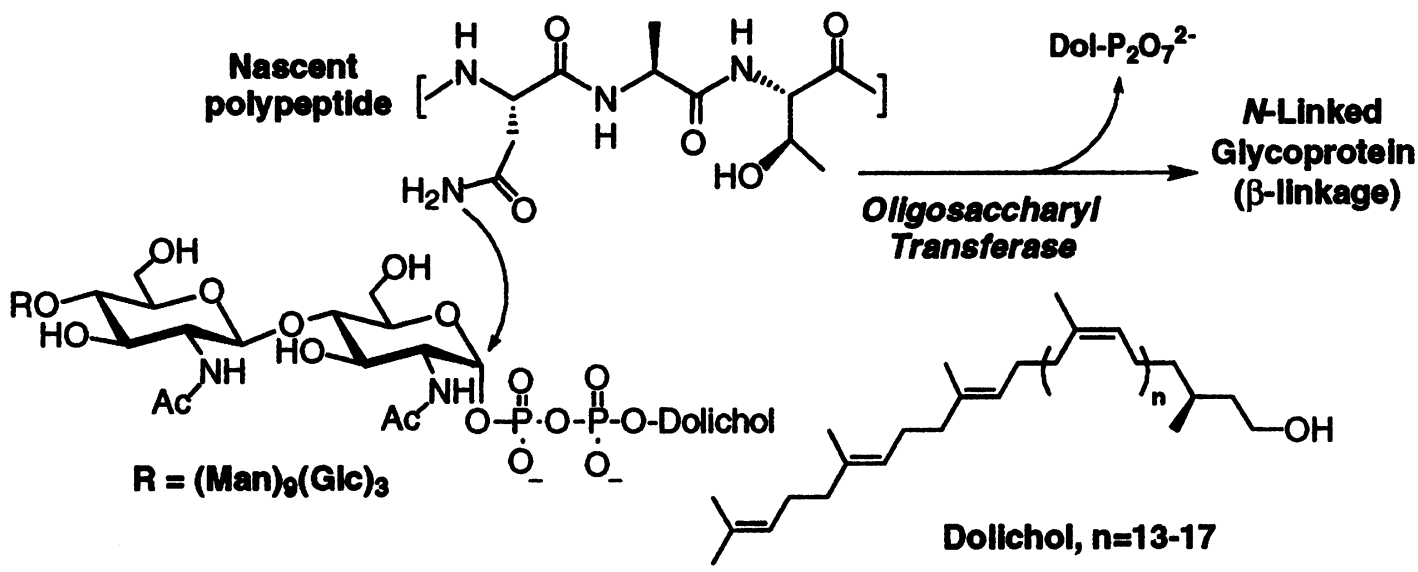

Fig. 1 Reaction catalyzed by oligosaccharyl transferase.

The ability of oligosaccharyl transferase to recognize and glycosylate short peptidyl substrates has facilitated investigation of the structural requirements for glycosylation. Specifically, peptide analogs which contain the recognition elements of the consensus sequence, but which are constrained to specific conformations, such as $\beta$-turns or an Asx-turn, have been designed, synthesized, and analyzed for both solution state structure as well as proficiency in a standard glycosylation assay [10,11]. The parallel structural and kinetic analysis of these compounds has provided insight into the role of peptide conformation in asparagine-linked glycosylation. Figure 2 illustrates the kinetic and structural data for a flexible glycosylation substrate $\mathbf{1}$ and the corresponding constrained analog $\mathbf{2}$. These studies revealed that those peptides that were constrained specifically to an Asx-turn motif through a side chain to main chain macrolactamization as in compound $\mathbf{2}$ were more competent substrates for the enzyme than the corresponding linear analog 1. It is noteworthy that the imposition of an Asx-turn constraint into the glycosyl acceptor peptide affords approximately a 10-fold improvement in the apparent $K_{\mathrm{M}}$ for oligosaccharyl transferase. In contrast, peptides satisfying the Asn-Xaa-Thr/Ser tripeptide sequence, but constrained to various $\beta$-turn motifs through the cyclic hexapeptide architecture, failed to show glycosyl acceptor properties [11]. Thus, these studies with conformationally constrained peptides have led to the proposal that the recognition motif for $N$-linked glycosylation involves an Asx-turn (Fig. 2).

\section{A}<smiles>CCCC(=O)NC(CC(N)=O)C(=O)NC(CC(C)C)C(=O)NC(C(=O)NC)C(C)O</smiles>

1

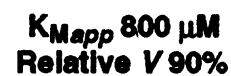

B

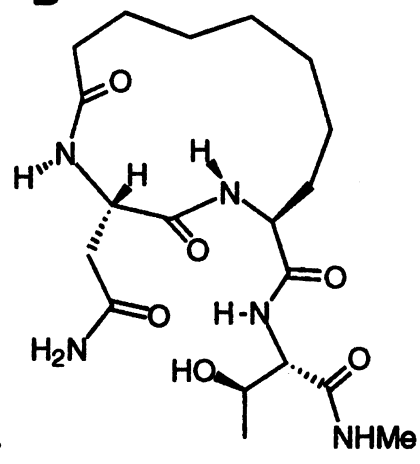

2

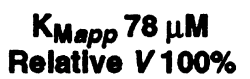

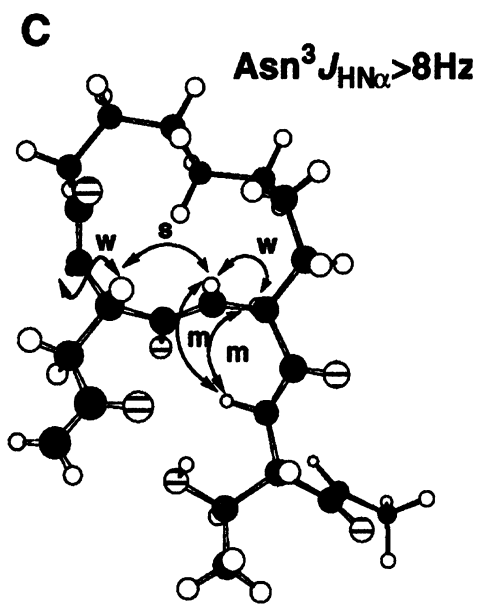

Fig. 2 Kinetic and structural analysis of oligosaccharyl transferase substrates. (A) 1-Linear Asn-Xaa-Thr tripeptide. (B) 2 -Corresponding substrate constrained to an Asx-turn. (C) Selected NMR data for solution state structural analysis of peptide 2 (strong (s), medium (m) and weak (w) ROEs as indicated were used in a simulated annealing protocol). The distinctive coupling constant for the asparagine $\mathrm{NH}-\alpha \mathrm{CH}$ was also used as a dihedral angle constraint in the structure determination. 
The Asx-turn is characterized by a 10-membered ring hydrogen bonding network in which the hydrogen bond acceptor is the carbonyl oxygen of the asparagine side chain and the hydrogen bond donor is the backbone amide proton of the residue at $(i+2)$ relative to asparagine (in this case the conserved hydroxy amino acid). This hydrogen-bonding motif is rather common in native proteins; approximately $18 \%$ of all asparagine and aspartic acid side chains appear to be involved in Asx-turns in proteins [12]. It should be noted that the homologous residue glutamine is never glycosylated and this apparent contradiction in reactivity may be explained by the distinct conformational preferences of this residue. Specifically, the carboxamide side chain of glutamine is seldom involved in short range hydrogenbonding interactions in proteins [12].

These conformational studies have formed a foundation for the development of a mechanistic model for the enzyme-catalyzed modification of the asparagine amide [13]. In this proposal, the unique hydrogen-bonding array provided by the Asx-turn is proposed to facilitate protonation of the carbonyl of the asparagine side chain while simultaneous, enzyme-mediated deprotonation at the nitrogen would effect the tautomerization of the carboxamide to an imidic acid or imidate species. Thus, tautomerization would afford a more nucleophilic species, which could in turn react with the electrophilic lipid-linked oligosaccharide (Fig. 3). This mechanism incorporates both the issues of specificity and reactivity as well as the absolute requirement for a hydroxy amino acid by integrating substrate structural requirements with participation of enzyme active site residues. Thus, the likelihood of an Asn-Xaa-Thr/Ser sequence to undergo glycosylation would be governed by the ability of each potential substrate to adopt an Asx-turn conformation within the active site of the enzyme (Fig. 3).
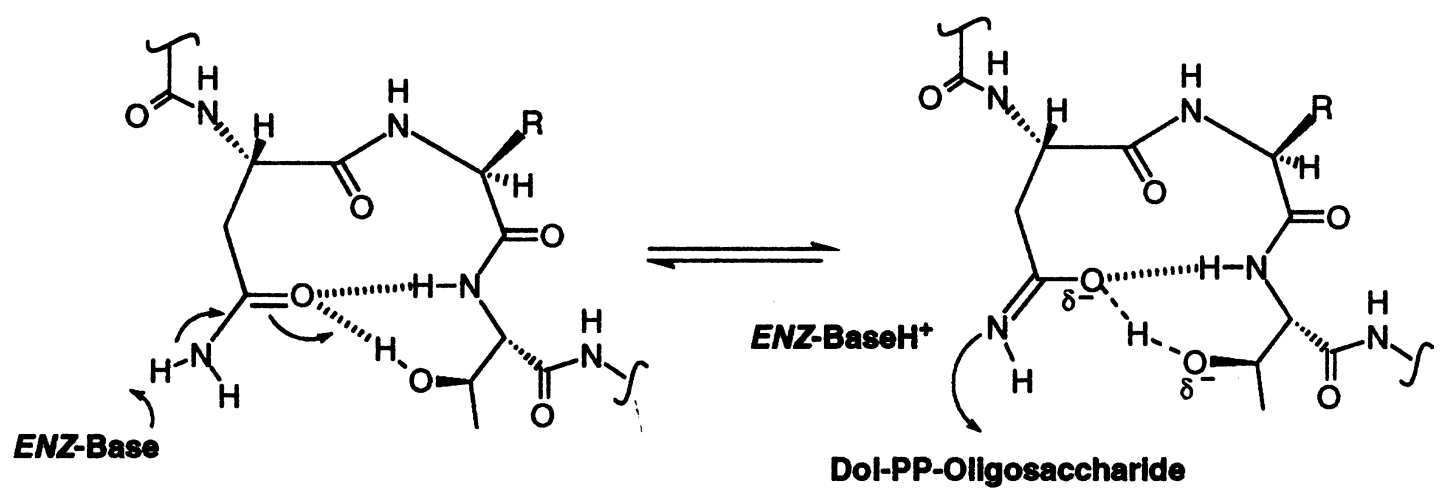

Fig. 3 A proposal for the mechanism of action of oligosaccharyl transferase.

\section{Development of potent and specific inhibitors of $\boldsymbol{N}$-linked glycosylation}

The conformational and mechanistic studies with oligosaccharyl transferase have led to a number of related developments in our ability to understand the glycosylation reaction. An important observation in the tripeptide analog studies was that the replacement of the carboxamide functionality of asparagine with the corresponding reduced amine species (from the residue 2,4-diaminobutanoic acid, Dab) resulted in a peptide with weak inhibitory properties. For example, as illustrated in Fig. 4, the linear tripeptide $\mathbf{3}$ shows inhibition of yeast oligosaccharyl transferase with a $K_{\mathrm{i}}$ of approximately $1 \mathrm{~mm}$. While the inhibitory properties of this peptide are rather weak, the kinetic analysis revealed that the mode of inhibition was clearly competitive. It was envisioned that improvement of the binding properties through further elaboration of the inhibitor structure could be achieved. The first critical feature of the inhibitor that was considered was the peptide backbone conformation. Since the Dab residue obviously lacks the critical carboxamide oxygen functionality that would promote formation of an Asx-turn, the Dab-Xaa-Thr motif was integrated into the constrained structure of inhibitor 4 (illustrated in Fig. 4) [10]. This constraint was observed to improve the $\mathrm{K}_{\mathrm{i}}$ considerably to afford a tripeptide with a $100 \mu \mathrm{M} K_{\mathrm{i}}$.

The further improvement of the peptide inhibitory properties was made possible by considering the fact that glycosylation substrates in vivo are not simple tripeptides, but rather extended sequences with numerous residues flanking the tripeptide sequon in both $C$ - and $N$-terminal directions. As the structure of oligosaccharyl transferase remains unknown it was not possible to use a structure based approach to 
<smiles>CNC(=O)[C@H](NC(=O)[C@H](CC(C)C)NC(=O)[C@H](CC[NH3+])NC(=O)c1ccccc1)[C@@H](C)O</smiles>

$K_{\mathrm{i}} \mathbf{1} \mathrm{mM}$

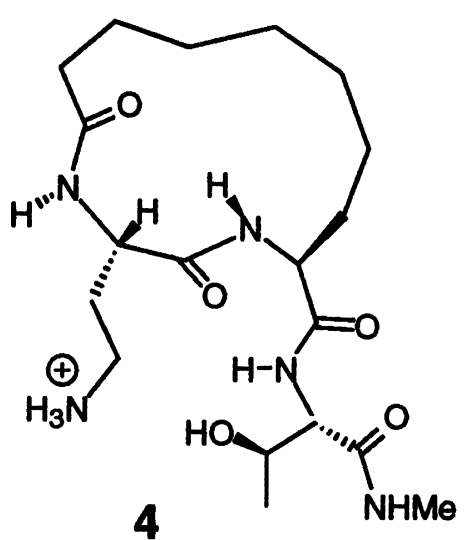

$K_{i} 100 \mu M$

Fig. 4 Competitive inhibitors of yeast oligosaccharyl transferase.

design the extended binding site. We therefore relied on statistical studies of the sequences of $N$-linked glycoproteins as a guide for defining the ideal residues adjacent to the threonine of the tripeptide sequon [14]. Thus, the next phase of inhibitor development included elongating the peptidyl structures to provide extended binding determinants for interaction with oligosaccharyl transferase. Modification of compound 4 to include flanking $N$-terminal residues was not synthetically straightforward with the macrocyclic structure; however, extension in the $C$-terminal direction was quite feasible. In order to facilitate these studies, an efficient solid phase synthesis of this class of inhibitors was developed.

The synthetic approach to the family of extended binding inhibitors of oligosaccharyl transferase is outlined in Fig. 5 [15]. The key features of the synthesis of $\mathbf{4}$ that needed remediating before a comprehensive evaluation of the utility of flanking residues could be carried out, included a fairly challenging macrolactamization step and the use of the synthetic, non-natural amino acid $\alpha$-aminodecanedioic acid (Add). Both of these features of the synthesis could be avoided if the critical cyclization was effected through the alkylation of a thiolate anion from the side chain of a central cysteine residue. The linear precursors to the constrained peptides could therefore be assembled by standard Fmoc-based solid phase synthesis protocols as illustrated in Fig. 5. The final key steps in the assembly of the inhibitors include orthogonal deprotection of the cysteine thiol protecting group (tributylphosphine) of the resin bound peptide, followed by treatment with the mild base tetramethylguanidine to affect thiolate alkylation by the 6-bromohexanoyl moiety. Using this chemistry, a family of 16 pseudohexpeptides was prepared for evaluation as inhibitors of oligosaccharyl transferase [16]. All the inhibitors included a key, cyclic tripeptide core with the Dab amino acid as well as a nitrophenylalanine residue at the $C$-terminal position to allow facile and accurate quantification of inhibitor concentrations. The principal variation amongst the inhibitor structures was the identity of the flanking Xaa and Yaa residues. In this study we chose to assess placement of a basic (lysine), acidic (glutamic acid), neutral (valine), and polar, uncharged (threonine) residue at each of the Xaa and Yaa positions. Additionally, the peptides were all examined with both a fungal (yeast) and mammalian (porcine liver) oligosaccharyl transferase (Fig. 5).

The results of inhibition studies on the family of 16 peptides that were prepared were quite dramatic. The estimated $K_{\mathrm{i}}$ values for each of the inhibitors for oligosaccharyl transferase from both yeast and porcine liver are reported in Fig. 6. $K_{\mathrm{i}}$ values range from high $\mu \mathrm{M}$ to low nM, with optimum binding observed when the Xaa-Yaa residues are Val-Thr ( $K_{\mathrm{i}} 25 \mathrm{~nm}$ for yeast OT and $30 \mathrm{~nm}$ for porcine liver OT). In all cases, placement of the basic residue, lysine, at either position Xaa or Yaa, significantly deteriorates binding. The results from these studies are in remarkable agreement with the statistical analyses of Gavel and von Heijne [14] in their systematic study of peptide sequences of known glycoproteins in the protein literature. For example, at position Xaa, the most frequently observed residue is valine, and indeed the same residue was found as optimum in the inhibitor study. Similarly, the least frequently observed residue is lysine, and it is this residue that is found in this position in the poorest of the inhibitors (Fig. 6). 
Fmoc-Dab(Boc)-Cys(StBu)-Thr(tBu)-Xaa-Yaa-Nph

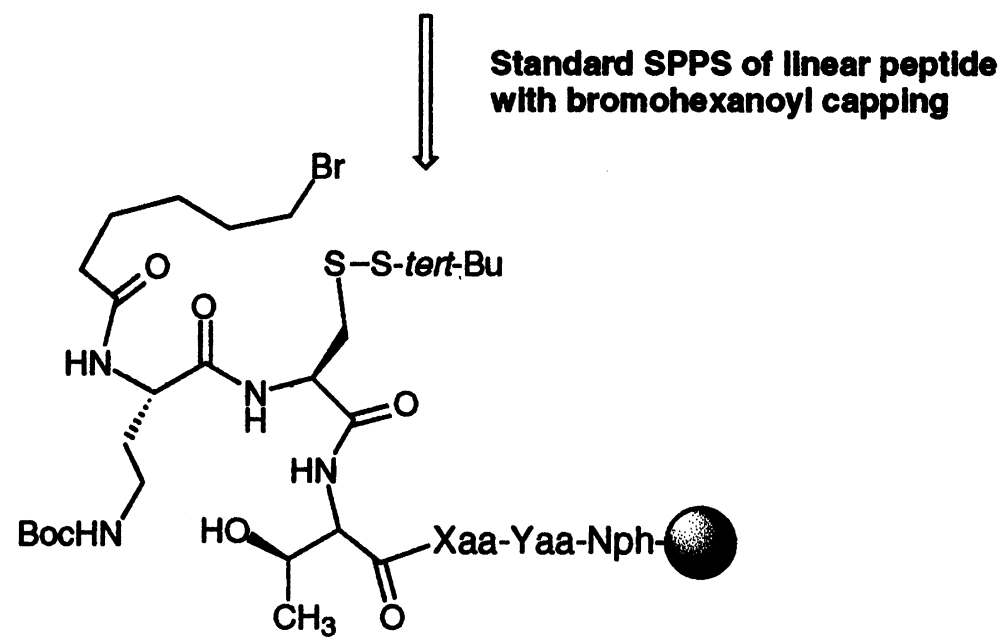

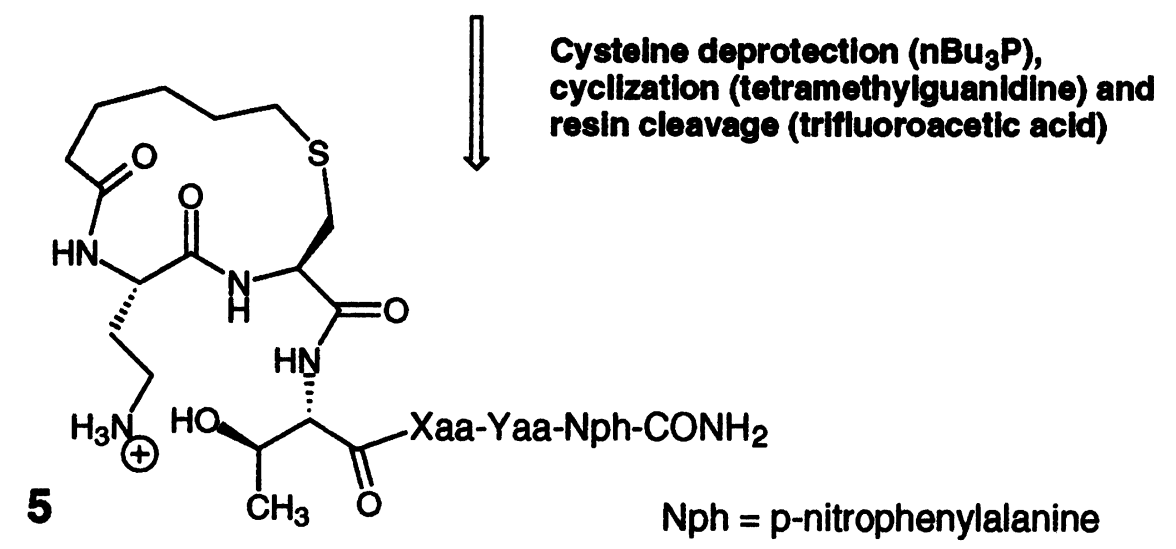

Fig. 5 Solid phase synthesis of extended binding inhibitors.

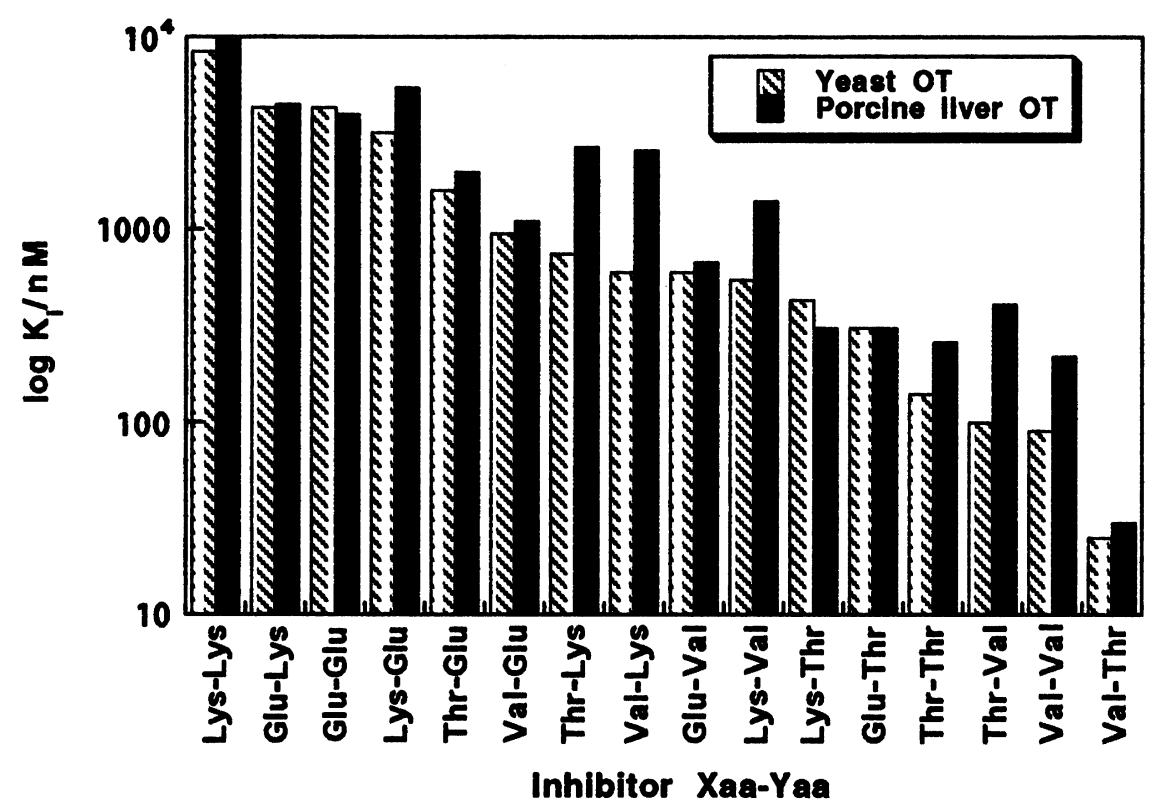

Fig. 6 Systematic evaluation of the inhibitory properties of the family of pseudohexapeptides related to peptide $\mathbf{5}$. Residues Xaa and Yaa are varied as shown on the $x$-axis. 
An important feature of the studies concerns the efficacy of the inhibitors with the two different oligosaccharyl transferase preparations. The detailed genetic analysis of oligosaccharyl transferase from a number of different species reveals a complex oligomeric architecture that appears to be highly conserved throughout eukaryotic evolution [5]. It would therefore be anticipated that the cyclic tripeptide core would exhibit little species selectivity. However, this situation may not prevail with the extended binding determinants because there is less evolutionary pressure for the residues that interact with these determinants to be conserved. Indeed we observed that in some cases (e.g. Xaa-Yaa $=$ Thr-Lys and ValLys) there was about a four-fold difference in the inhibition potency. These preliminary data suggest that it may be possible to design species specific inhibitors of $N$-linked glycosylation by exploiting the extended binding determinants.

While the peptidyl inhibitors that have been described are very potent for in vitro analyses of oligosaccharyl transferase activity, studies with these compounds in whole cell assays of $N$-linked glycosylation reveal the compounds to be ineffectual. This is presumably because the peptides are too polar and strongly solvated to be able to pass passively across the external cellular membrane and the ER membrane to gain access to the enzyme in its native cellular location in the lumen of the ER (Fig. 7).

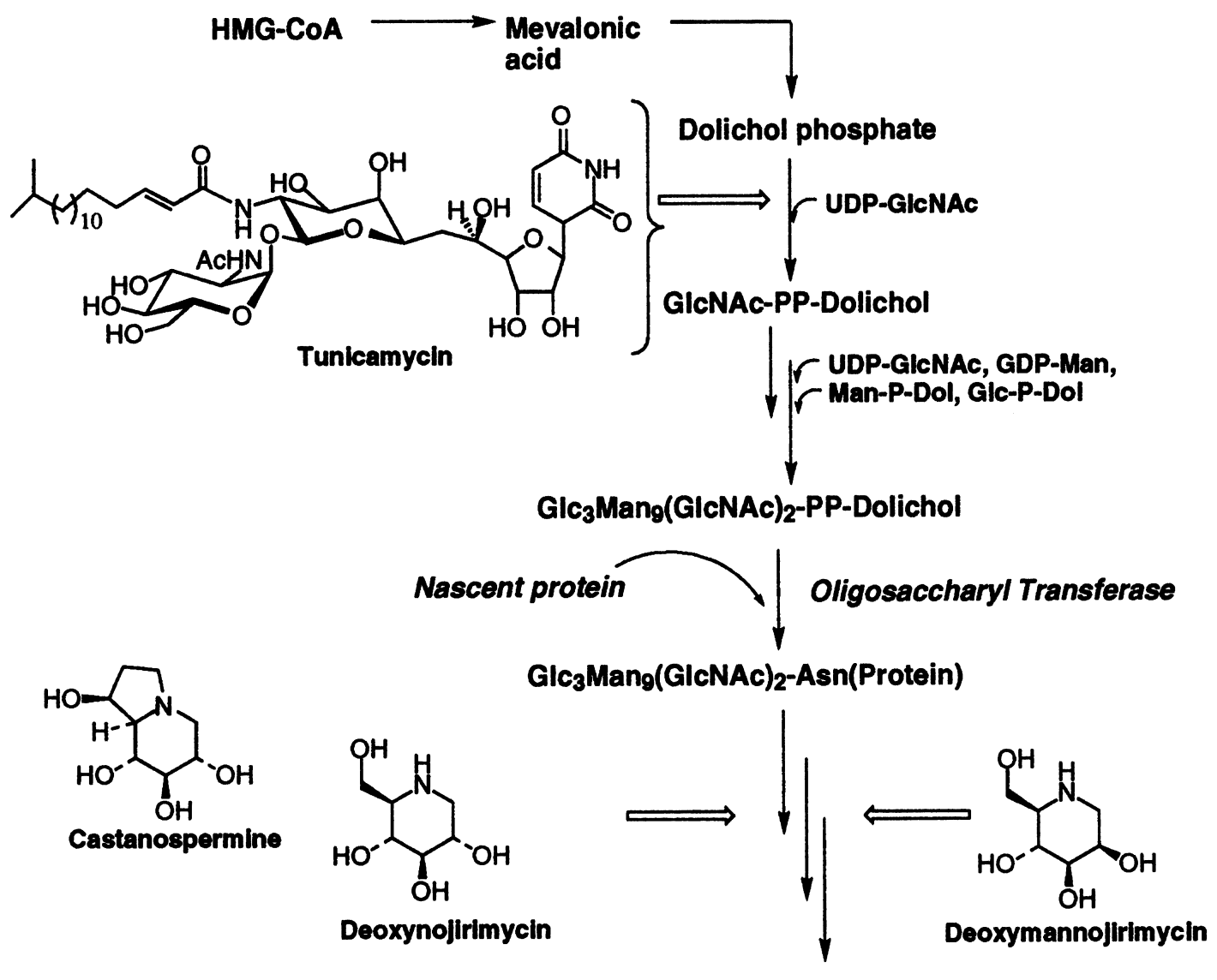

MATURE GLYCOPROTEIN

Fig. 7 Survey of glycosylation inhibitors.

A current focus in our laboratories is the manipulation of the inhibitor structures to improve cellular permeability properties. These studies are timely because a specific cellular tool for inhibiting asparaginelinked glycosylation does not exist. Currently, the only inhibitor of $N$-linked protein glycosylation that demonstrates activity at a practical concentration is the microbial product tunicamycin [17]; however, the effect of tunicamycin on protein glycosylation is neither specific nor immediate because it functions by inhibiting the first step in the assembly of the oligosaccharide donor (Dol-P-P-(GlcNAc $\left.)_{2}-(\mathrm{Man})_{9}-(\mathrm{Glc})_{3}\right)$ 
essential in the formation of all asparagine-linked glycoproteins as illustrated in Fig. 7. Furthermore, use of tunicamycin requires several cell cycles before the supply of the donor is sufficiently depleted to arrest glycosylation. Catanospermine and members of the nojirimycin family of glycosylation inhibitors are also frequently used to probe the biological significance of cellular glycosylation [18]. However, like tunicamycin, these natural products do not function by inhibiting oligosaccharyl transferase directly; the molecular targets for these inhibitors are the processing enzymes that function to modify the structure of the triantennary tetradecasaccharide once it has been transferred to a nascent protein.

\section{Conformational consequences of asparagine-linked glycosylation}

An additional area of current interest concerns the study of the conformational consequences of asparaginelinked glycosylation. It is now evident that the co-translational timing of $N$-linked glycosylation can influence the conformational dynamics of the newly biosynthesized peptide proximal to the glycosylation site and that this structural modulation can potentially affect the subsequent folding of the glycoprotein product. The experimental evidence for this fact is derived from three types of experiments. First, the expression of glycosylated eukaryotic proteins in prokaryotic systems, which lack the glycosylation machinery, frequently results in the formation of aggregated or misfolded proteins. Second, the experiments involving deletion of glycosylation sites via site-directed mutagenesis have demonstrated the requirement of numerous $N$-linked glycosylation sites for proper protein folding. Finally, protein expression in the presence of the glycosylation inhibitor tunicamycin often results in misfolded proteins that fail to be secreted in a normal native conformation. It is not surprising that the large, hydrophilic carbohydrate moiety has a profound impact on the backbone structure of a polypeptide; numerous examples of glycosylation-mediated conformational change for both proteins and small peptides are available [2]. However, the structural details of the glycosylation-induced conformational changes have yet to be defined.

The experiments carried out in this laboratory focus on the study of polypeptide segments that are patterned after glycosylation sites in native proteins. One peptide that has been studied in considerable detail by complementary spectroscopic methods is derived from haemagglutinin from influenza A virus. These homotrimeric viral coat proteins are generally heavily glycosylated. For example, the 1968 Hong Kong X31 strain haemagglutinin has six glycosylated Asn-Xaa-Ser/Thr sites and it is estimated that 20\% of the glycoprotein weight is carbohydrate [19]. A number of the glycosylation sites within each of the monomer units of haemagglutinin are strongly conserved and are found at $\beta$-turn motifs in the final folded protein structure [19]. We have carried out both fluorescence [20] and nuclear magnetic resonance (NMR) studies $[21,22]$ on the segment of polypeptide that includes Asn-285. This glycosylated asparagine is found at the $(i+2)$ position of a $\beta$-turn in the folded protein.

Initial experiments with this system included fluorescence resonance energy transfer (FRET) studies of appropriately derivatized peptides and glycopeptides representing the Asn-285 peptide sequence. Glycopeptides were prepared in vitro by oligosaccharyl transferase mediated glycosylation using the truncated glycosyl donor dolichylpyrophosphorylchitobiose [20]. These studies revealed that modification with the disaccharide had a significant impact on the polypeptide conformation in aqueous media. FRET studies suggested that the structure of the nonglycosylated peptide was fairly extended, as evidenced by the large interfluorophore distance. In contrast, the structure of the corresponding glycosylated peptide was significantly more compact with an interfluorophore distance of about $7 \AA$. The changes in the interfluorophore distances were consistent with a switch from an extended conformation to a compact turn conformation, strongly suggesting that glycosylation induced the adoption of a folded motif.

The results from the fluorescence studies encouraged us to pursue a detailed NMR analysis of similar systems with the goal of achieving greater insight into the molecular detail of the glycosylation induced conformational changes [22]. In this case the need for greater quantities of material necessitated the chemical synthesis of glycopeptides for study. Glycopeptide synthesis was effected using a modification of the methods developed previously [23,24]. Specifically, glycopeptides were prepared from the corresponding peptides in which an allyl-protected aspartic acid was substituted for the asparagine in the parent sequence. At the completion of peptide synthesis, the allyl group was deprotected using a 
palladium catalyst and then the glycosyl group was installed by coupling the resin-bound peptide with the appropriate unprotected glycosyl amine. The tendency for allyl esters of aspartic acid to undergo facile succinimide formation during the peptide synthesis necessitated the use of the 2-hydroxy-4methoxybenzyl (Hmb) amide protecting group [25] on the glycine adjacent to the aspartic acid during the peptide synthesis.

Ac-Orn-Ile-Thr-Pro-Asn ${ }^{285}$-Gly-Thr-Trp-Ala- $\mathrm{NH}_{2}$

Ac-Orn-Ile-Thr-Pro-Asn (GlcNAc) ${ }_{2}$-Gly-Thr-Trp-Ala- $\mathrm{NH}_{2}$

The 2D NMR studies of peptide 6 and the corresponding glycopeptide 7 provided evidence that glycosylation with chitobiose, a disaccharide representing the first two $\beta$ [1-4] linked $N$-acetylglucosamine residues of the native tetradecasaccharide, induces the formation of a compact type I $\beta$-turn. The extended structure of the nonglycosylated peptide (6-Fig. 8A) was found to resemble the Asx-turn which had been proposed to be important for oligosaccharyl transferase recognition. The more compact structure of 7 (Fig. 8B), previously suggested by the FRET studies of the glycosylated derivative and supported by the NMR analysis, strongly resembled the type I $\beta$-turn found in the native protein. In the NMR analysis, NOE data were incorporated as distance restraints into a simulated annealing protocol to produce the illustrated structures (Fig. 9A and B). The structure of the glycopeptide was remarkably similar to the structure found in the fully folded glycoprotein. Since approximately $30 \%$ of all glycosylation sites occur at sites which fold ultimately to $\beta$-turns [26], this model peptide provides a relatively 'general' context for the study of the conformational implications of $N$-linked glycosylation.

A

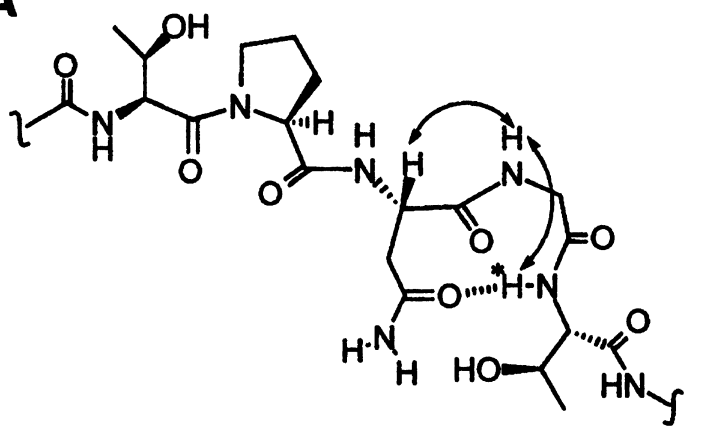

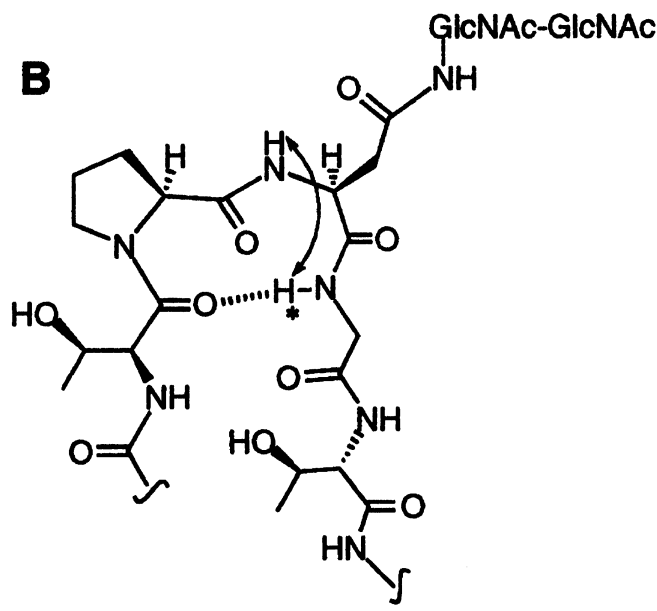

Fig. 8 Several strong key NOEs distinguish between an Asx and $\beta$-turn structure. Starred amide protons have a low variable temperature coefficient, suggesting the presence of intermolecular hydrogen bonds.

Despite the dramatic change in peptide conformation that was observed upon protein glycosylation, no obvious specific interactions between this peptide and the chitobiose moiety were observed in the NMR analysis. This suggests that the conformational changes observed after peptide glycosylation may be driven by either simple steric effects or by an alteration of the local water structure by the attached carbohydrate rather than by the formation of specific non-covalent interactions.

To further probe the nature of the interaction between carbohydrate and peptide, parallel NMR studies were performed on a family of glycopeptides in which key molecular elements of the sugar, specifically the C-2 $\mathrm{N}$-acetamido groups, were modulated (Fig. 10) [21].

The average structure of each glycopeptide in aqueous media was determined by examining NOE data and ${ }^{3} \boldsymbol{J}_{\mathrm{HN} \alpha}$ values. To complement these structural studies, the conformational stability of the glycopeptides was assessed with ${ }^{13} \mathrm{C}_{1}$ relaxation values. These analyses revealed that chitobiose plays a unique role in modulating peptide conformation; no other saccharide appears to induce the native $\beta$-turn structure. Additionally, the chitobiose derivatized peptide appears to be more rigid at the glycosylation 
A

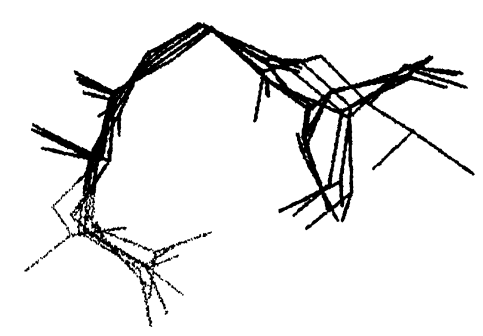

B

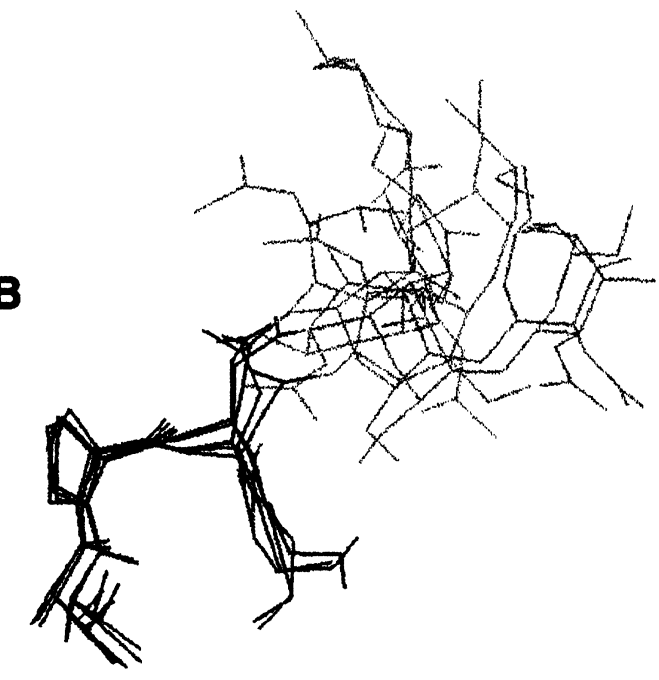

Fig. 9 Structures of (A) nonglycosylated and (B) glycosylated peptides derived from a simulated annealing procedure that incorporated all NOE data.

\section{Ac-Orn-Ile-Thr-Pro-Asn ${ }^{285}$-Gly-Thr-Trp-Ala-CONH 2 \\ Carbohydrate}<smiles>CCC1(C)OC(CO)C(O)C(O)C1N</smiles>

B-D-GICNAC

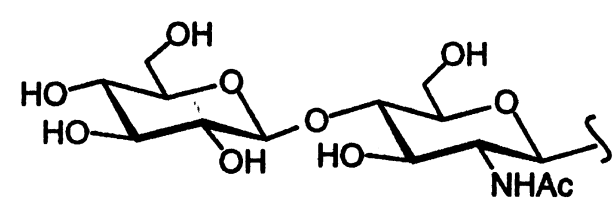

D-GIC-[ $[\beta(1,4)]-\beta-D-G I C N A C$

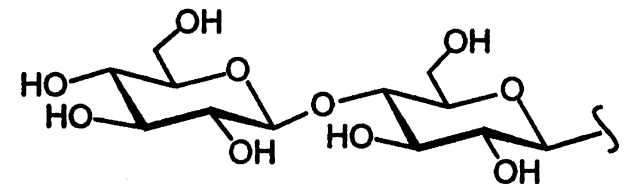

Cellobiose

Fig. 10 Summary of glycopeptides studied by NMR.

site than the other derivatives. The NMR derived structures of glycopeptides modified with cellobiose and D-Glc- $[\beta(1,4)]-\beta$-D-GlcNAc suggest that the $N$-acetyl group proximal to the peptide is critical for the induction of $\beta$-turn formation (Fig. 11). Cellobiose, which lacks the proximal $N$-acetyl group, promotes a more extended peptide conformation (Fig. 11A), while D-Glc- $[\beta(1,4)]-\beta-D-G l c N A c$ (Fig. 11B) promotes a $\beta$-turn structure. Since no evidence for a specific interaction between the carbohydrate and peptide was found, it would appear that the structural modulation is most likely effected through non-specific steric interactions. Surprisingly, the addition of an $\mathrm{N}$-acetyl group may restrict rotation around the glycosidic bond, thereby promoting a more rigid carbohydrate conformation.

\section{CONCLUSIONS}

Asparagine-linked glycosylation is one of the most complex enzyme-catalyzed protein modification reactions. Moreover, the co-translational timing of this transformation implies that glycosylation plays a 
A

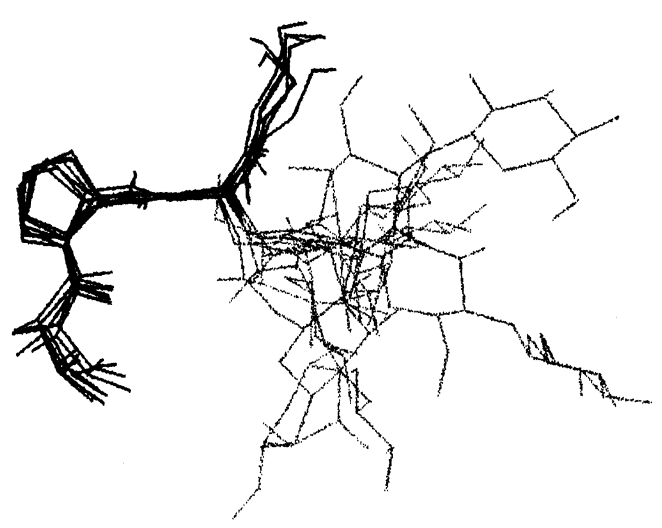

B

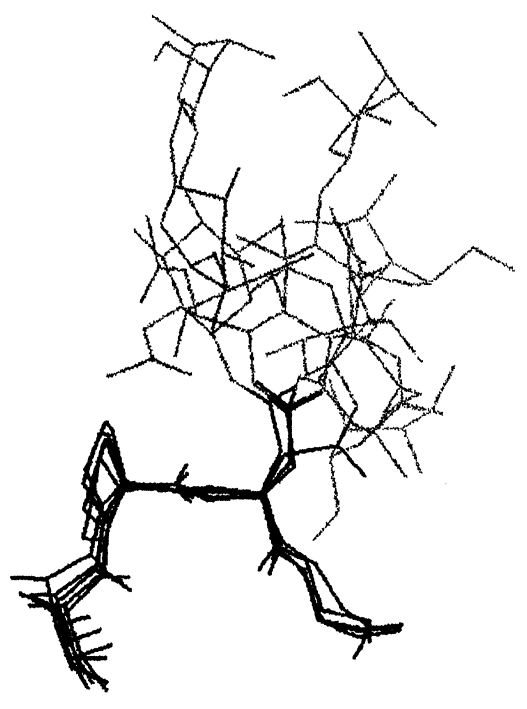

Fig. 11 Structures of (A) Cellobiose modified glycopeptide and (B) Peptide modified with Glc-[ $\beta(1,4)]-\beta-D-$ GlcNAc. Structures are derived from a simulated annealing procedure that incorporated all NOE data.

unique role in the protein biosynthesis process. While remarkable progress has been made towards understanding the molecular players in the glycosylation reaction, there remain many obstacles to overcome before a clear mechanistic picture of the enzyme emerges. In the next phase of research the complementary application of state-of-the-art bioorganic and biophysical methods, together with the tools of contemporary molecular biology will contribute to a more satisfactory description of this fascinating enzyme along with its many biological implications.

\section{ACKNOWLEDGEMENTS}

This research was supported by the National Institutes of Health (GM 39334).

\section{REFERENCES}

1 A. Varki. Glycobiology 3, 97-130 (1993).

2 P. M. Rudd, R. A. Dwek. Crit. Rev. Biochem. Mol. Biol. 32, 1-100 (1997).

3 B. Imperiali. Acc. Chem. Res. 30, $452-459$ (1997).

4 R. Kornfield, S. Kornfield. Ann. Rev. Biochem. 54, 631-664 (1985).

5 S. Silberstein, R. Gilmore. FASEB J. 10, 849-858 (1996).

6 P. Vandensteen, P. M. Rudd, R. A. Dwek, G. Opdenakker. Crit. Rev. Biochem. Mol. Biol. 33, 151-208 (1998).

7 P. T. Englund. Ann. Rev. Biochem. 62, 121-138 (1993).

8 I. Nilsson, G. von, Heijne. J. Biol. Chem. 268, 5798-5801 (1993).

9 R. D. Marshall. Ann. Rev. Biochem. 41, 673 (1972).

10 B. Imperiali, J. R. Spencer, M. D. Struthers. J. Am. Chem. Soc. 116, 8424-8425 (1994).

11 B. Imperiali, K. L. Shannon, K. W. Rickert. J. Am. Chem. Soc. 114, 7942-7944 (1992).

12 E. N. Baker, R. E. Hubbard. Prog. Biophys. Molec. Biology 44, 97 (1984).

13 B. Imperiali, K. L. Shannon, M. Unno, K. W. Rickert. J. Am. Chem. Soc. 114, 7944-7945 (1992).

14 Y. Gavel, G. vonHeijne. Protein Eng. 3, 433-442 (1990).

15 T. L. Hendrickson, J. R. Spencer, M. Kato, B. Imperiali. J. Am. Chem. Soc. 118, 7636-7637 (1996).

16 C. Kellenberger, T. Hendrickson, B. Imperiali. Biochemistry 36, 12 554-12559 (1997). 
17 D. A. Elbein. Trends Biol. Sci. 6, 291-293 (1981).

18 D. A. Elbein. FASEB J. 5, 3055-3063 (1991).

19 I. A. Wilson, R. C. Ladner, J. J. Skehel, D. C. Wiley. Biochem. Soc. Trans. 11, 145-147 (1981).

20 K. W. Rickert, B. Imperiali. Chem Biol. 2, 751-759 (1995).

21 S. E. O’Connor, B. Imperiali. Chem. Biol. 5, 427-437 (1998).

22 S. E. O'Connor, B. Imperiali. J. Am. Chem. Soc. 119, 2295-2296 (1997).

23 S. A. Kates, B. G. delaTorre, R. Eritja, F. Albericio. Tetrahedron Lett. 35, 1033-1034 (1994).

24 S. T. Cohen-Anisfeld, P. T. Lansbury. J. Am. Chem. Soc. 115, 10531-10 537 (1993).

25 J. Offer, M. Quibell, T. Johnson. J. Chem. Soc. Perkin Trans. 1, 175-182 (1996).

26 J. J. Beintema. Bioscience Rep. 6, 709-714 (1986). 